\title{
Retrieving Information From a Hierarchical Plan
}

\author{
Darryl W. Schneider and Gordon D. Logan \\ Vanderbilt University
}

\begin{abstract}
Plans give structure to behavior by specifying whether and when different tasks must be performed. However, the structure of behavior need not mirror the structure of the plan. To investigate this idea, the authors studied how plan information is retrieved in the context of a novel sequence-position cuing procedure, wherein subjects memorize two task sequences, then perform trials on which they are randomly cued to perform a task at one of the serial positions in a sequence. Several empirical effects were consistent with retrieval from a hierarchically structured representation (but not a non-hierarchical representation), including large sequence-repetition benefits, position-repetition benefits only for sequence repetitions, and a lack of robust task-repetition benefits. The data were successfully modeled by assuming that retrieval was time-consuming, susceptible to priming, cue-dependent, structurally constrained, and token-specific. In tandem, the empirical data and modeling work provide deeper insight into the representation of and access to information in memory that comprises a plan for guiding behavior.
\end{abstract}

Keywords: hierarchical representation, plan, task sequence, memory retrieval

A noteworthy feature of the human cognitive system is its ability to coordinate the performance of multiple tasks, giving rise to complex patterns of behavior. The means by which such coordination is possible have been explored extensively in cognitive psychology, leading to the idea that behavior is often guided by a plan - an internal representation that organizes thought and action (Miller, Galanter, \& Pribram, 1960; Schank \& Abelson, 1977). Plans give structure to behavior by specifying whether and when different tasks must be performed. By understanding how plans are represented and accessed, it may be possible to understand how they control behavior (Morris \& Ward, 2005) and how planned behavior can be impaired (Cooper \& Shallice, 2000; Duncan, 1986; Humphreys \& Forde, 1998).

Plans can be represented in several ways. In this article, we focus on two broad classes of representation: hierarchical and non-hierarchical. A hierarchical plan is one in which information is represented at two or more levels. For example, a daily to-do list may be divided into morning and afternoon, with a different list of tasks associated with each time period. A non-hierarchical plan-in its simplest form-is one in which information is represented at a single level. For example, a to-do list may be a single list of tasks to be accomplished by the end of the day. In both cases, the plans could be associated with overarching goals, with each task being a component of a larger task or task performance cumulating toward a specific outcome. However, tasks could also be relatively independent and non-cumulative, as might be the case with tasks on a to-do list. We chose to study non-cumulative plans

Darryl W. Schneider and Gordon D. Logan, Department of Psychology, Vanderbilt University.

This research was supported by National Science Foundation Grants BCS 0446806 and BCS 0646588 to Gordon D. Logan. We thank Julie Delheimer for running the experiment.

Correspondence concerning this article should be addressed to Darryl W. Schneider, Department of Psychology, Vanderbilt University, Nashville, TN, 37203. E-mail: darryl.schneider@vanderbilt.edu (in the form of arbitrary task sequences) in the present investigation because they are more amenable to our experimental procedure and they have yielded interesting findings in recent work (Logan, 2004, 2007; Schneider \& Logan, 2006).

Plans can be accessed in several ways. A common method is to access the elements of a plan in a serial manner. For example, the tasks on a to-do list could be performed one after another in the order in which they appear on the list. This mode of access would yield serially ordered behavior that mirrors the serial structure of the list. However, serially ordered behavior may not reflect a serial, non-hierarchical representation (e.g., an associative chain) in memory (Lashley, 1951). The structure of behavior need not be isomorphic to the structure of the representation underlying that behavior. Indeed, there are many ways that a hierarchical representation can be interpreted to yield serial behavior (Greeno \& Simon, 1974). A major goal of the present study is to demonstrate that a hierarchical representation can produce behavior whose structure does not mirror that of the representation.

The relationship between the structure of behavior and the structure of the underlying representation or processes has recently received attention in the domain of routine sequential action (i.e., the study of everyday, multi-step tasks such as making a cup of coffee). Botvinick and Plaut (2004) noted that "the majority of existing models continue to assume that the hierarchical structure of sequential behavior is directly reflected in the structure of the processing system, as a hierarchy of nodes or schemas" (p. 396). Those authors challenged this assumption-and models that embody the assumption (e.g., Cooper \& Shallice, 2000)—by demonstrating that non-hierarchical, distributed representations within a connectionist network were sufficient to model routine sequential action, sparking a debate about whether sequential behavior requires a hierarchically structured representation (Botvinick \& Plaut, 2006; Cooper \& Shallice, 2006a, 2006b).

The debate concerning routine sequential action can be viewed as addressing two issues. The first is whether the structure of behavior and the structure of the underlying representation are 
isomorphic; the second is whether the representation is hierarchically structured. In the present study, we were able to focus on the second issue by precluding isomorphism between behavior and representation. This was accomplished by studying whether a pattern of behavior could be explained in terms of retrieval of information from a hierarchical or a non-hierarchical representation, even though the structure of behavior mirrored neither representation.

To elaborate, let us return to the example of the to-do list with different tasks associated with the morning and afternoon. Recall that such a list can be represented hierarchically (by having separate lists for morning and afternoon) or non-hierarchically (as a single list for the entire day). Imagine that instead of performing the tasks on the list in serial order, a person was asked to perform the second task from the afternoon, followed by the fourth task from the morning, then the first task from the afternoon, and so forth. If the person performed the tasks in random order, then the structure of the resultant pattern of behavior would not mirror the structure of either the hierarchical representation or the nonhierarchical representation described above. Regardless of the structure of the representation, there would be no isomorphism between behavior and representation. However, the representation of the tasks would still be structured, and the cues to perform one task or another would require access to this structured representation. Understanding how this access occurs-and determining whether it involves a hierarchical or a non-hierarchical representation-requires taking our to-do list example into the laboratory and developing a procedure that allows us to control when and how information from a plan is accessed.

\section{The Sequence-Position Cuing Procedure}

We developed a novel cuing procedure to study non-isomorphic behavior arising from a structured representation. In the sequenceposition cuing procedure, subjects memorize two task sequences and then perform trials on which they are randomly cued to perform a task at one of the serial positions in a sequence. For example, in the experiment reported below, subjects performed origin (living vs. nonliving) and size (small vs. large) tasks on the referents of target words. These tasks were organized into different sequences labeled alpha and beta, such that both tasks occurred equally often in each sequence, but at different serial positions (e.g., alpha $=$ origin, origin, size, size; beta $=$ size, origin, origin, size). After memorizing and practicing the alpha and beta sequences, subjects completed trials on which sequence-position cues were presented. A cue such as alpha three indicated that the third task in the alpha sequence - the size task-should be performed, whereas the cue beta two indicated that the second task in the beta sequence-the origin task-should be performed. Cues were selected randomly, such that the series of tasks performed across trials did not consistently correspond to either of the memorized task sequences (i.e., they were not isomorphic). Thus, to perform accurately in the experiment, subjects had to retrieve the identity of the relevant task on each trial by using the presented cue to access a structured representation in memory.

The question of interest is whether the structure of the representation underlying retrieval in the sequence-position cuing procedure is hierarchical or non-hierarchical. An example of each type of representation is depicted in Figure 1 for the aforementioned alpha and beta sequences. Ovals in the figure represent knowledge units (see also Anderson \& Matessa, 1997) composed of sequence, position, and/or task information (as indicated by arrows). We assume that the knowledge units exist only in the context of the experiment, which is why "Experiment" appears at the top of each representation. Beyond that, the representations differ in terms of the type and organization of knowledge units. In the hierarchical representation in Figure 1A, there are higher level units for each sequence (alpha and beta) and nested, lower level units with position and task information. In the non-hierarchical representation in Figure 1B, there is only a single level of units with sequence, position, and task information. Successful retrieval involves ultimately accessing the knowledge unit that contains the relevant task identity.

How might retrieval occur from each representation? For the hierarchical representation, if one follows the retrieval schemes proposed in previous research (e.g., Anderson, Bothell, Lebiere, \& Matessa, 1998; Anderson \& Matessa, 1997; Collard \& Povel, 1982; Greeno \& Simon, 1974; Rosenbaum, Kenny, \& Derr, 1983; see also Dixon, 1987), the sequence unit would have to be accessed before information about task identity could be retrieved from a nested position unit. For example, the cue alpha three would require accessing the alpha sequence unit, followed by the nested three position unit to identify the relevant task as the size task (see Figure 1A). In the case of the non-hierarchical representation, retrieval could occur in a single step by using the sequence and position parts of the cue as joint cues for accessing a unique sequence-position unit. For example, the cue alpha three would retrieve the size task directly from the alpha three unit. In this sense, the cues for a non-hierarchical representation are functionally equivalent to arbitrary task cues (i.e., cues that are not meaningfully associated with tasks; e.g., letters, colors, shapes, etc.) used in many task-switching studies, and the mode of retrieval is a variant of compound-cue retrieval (Logan \& Bundesen, 2004; Ratcliff \& McKoon, 1988; Schneider \& Logan, 2005).

\section{Experiment Overview}

To distinguish between retrieval from hierarchical and nonhierarchical representations in a situation in which behavior does not mirror either representation, we conducted a sequence-position cuing experiment in which we examined the effects of several variables on performance, focusing primarily on response time (RT) as the dependent measure. The first variable was cuing condition: The onsets of the sequence and position parts of the cue were manipulated by varying which parts of the cue were available during a cue-target interval (CTI). The different cuing conditions are illustrated in Figure 2. In the sequence cuing condition, only the sequence part of the cue (e.g., alpha) was available during the CTI; the position part (e.g., three) was presented at target onset. In the sequence-position cuing condition, both parts of the cue (e.g., alpha three) were available during the CTI. By manipulating the CTI, we could measure the time course of retrieval in each cuing condition.

The remaining variables were different types of transitions that could occur across trials. Sequence transitions were either sequence repetitions (e.g., alpha $\rightarrow$ alpha) or sequence switches (e.g., beta $\rightarrow$ alpha). Position transitions were either position repetitions (e.g., three $\rightarrow$ three) or position switches (e.g., one $\rightarrow$ 


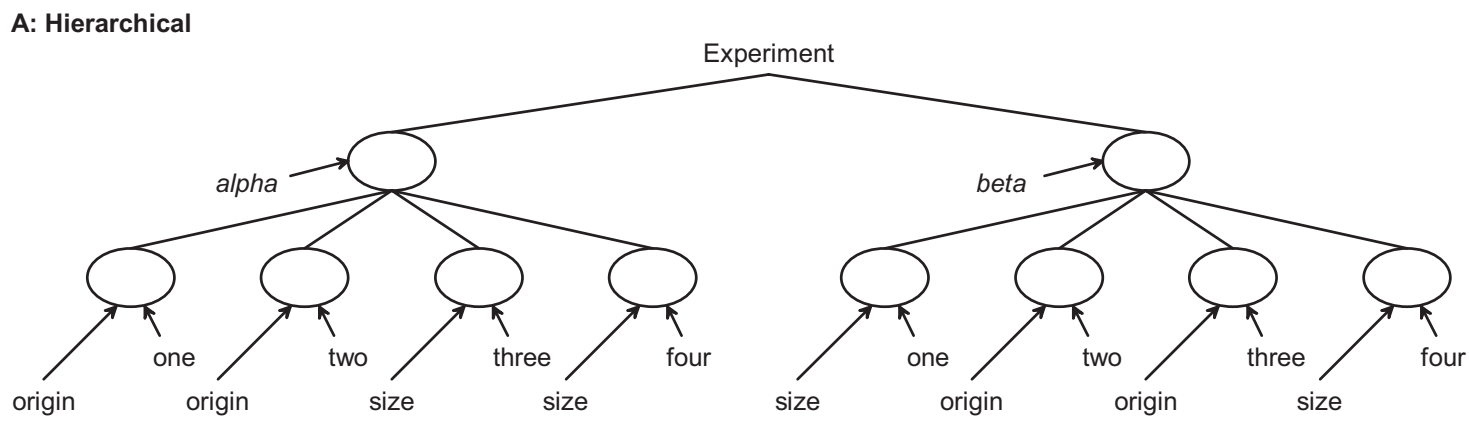

B: Non-Hierarchical

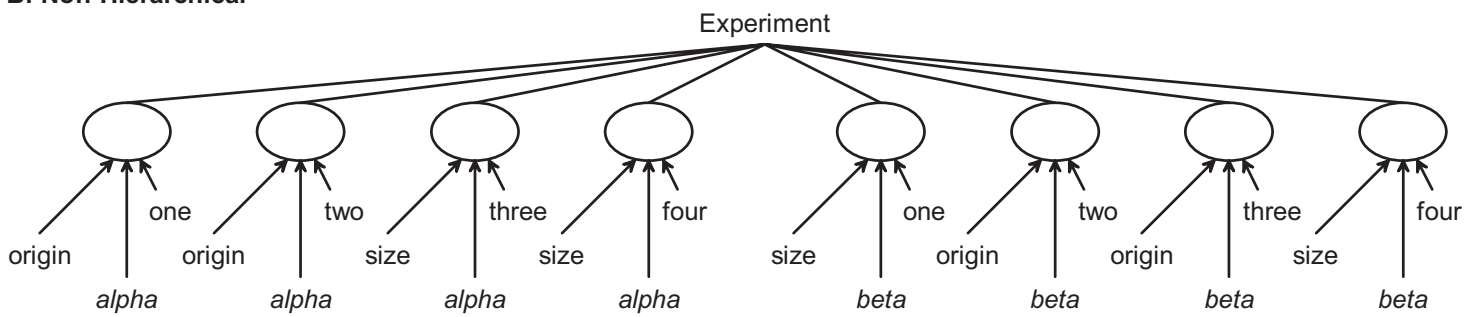

Figure 1. Hierarchical and non-hierarchical representations for sequences (labeled alpha and beta) composed of origin and size tasks at specific serial positions. Ovals represent knowledge units composed of sequence, position, and/or task information (as indicated by arrows). Modeled on Figure 2 in Anderson and Matessa (1997, p. 732).

three). Task transitions were either task repetitions (e.g., origin $\rightarrow$ origin) or task switches (e.g., size $\rightarrow$ origin). Note that position transition is partially confounded with task transition (for sequence repetitions, position repetitions are always task repetitions). The final type of transition was based on the form of the cue: Both parts of the cue could appear in either word form (e.g., alpha three) or letter-digit form (e.g., $\alpha 3$ ). Form transitions were either form repetitions (e.g., word form $\rightarrow$ word form) or form switches (e.g., letter-digit form $\rightarrow$ word form). These different types of transitions are expected to have different effects on performance as a function of CTI in each cuing condition. However, the critical question is whether these effects will vary as a function of the structure of the representation (hierarchical vs. non-hierarchical) that could be guiding retrieval.

\section{Predictions}

Hierarchical representation. If information is retrieved from a hierarchical representation, then there should be a benefit of accessing the same knowledge unit across trials. (A detailed explanation of how such a benefit could occur is presented later in this article.) This leads to the prediction of a sequence-repetition benefit, such that sequence repetitions are faster than sequence switches. Sequence-repetition benefits (sometimes referred to as sequence-switch costs) have been observed in several studies and are commonly interpreted as evidence of a hierarchical representation (e.g., De Jong, 1995; Lien \& Ruthruff, 2004; Luria \& Meiran, 2003; Schneider \& Logan, 2006; but see other considerations below). These benefits would be expected to decrease with CTI in each cuing condition because the sequence part of the cue is always presented before target onset, thereby allowing differences in the retrieval of sequence information to be absorbed during longer CTIs. However, the time course of retrieval should be much steeper in the sequence-position cuing condition than in the sequence cuing condition because in the former, both sequence and position units can be accessed during the CTI, whereas in the latter, only sequence units can be accessed during the CTI.

Position-repetition benefits would also be expected, but only for sequence repetitions. Recall that in a hierarchical representation, access to a sequence unit is required before a nested position unit can be accessed. A position repetition in the context of a sequence repetition would involve accessing the same knowledge unit, yielding a benefit. However, a position repetition in the context of a sequence switch would involve accessing a different knowledge unit (see Figure 1A), yielding no benefit.

Form transitions would modulate sequence- and positionrepetition benefits. Form repetitions would be expected to enlarge these benefits because the same cue would be used to access the same knowledge unit across trials, yielding form-repetition benefits restricted to sequence and position repetitions. Such formrepetition benefits are essentially cue-repetition benefits, which have been observed in several studies and interpreted as evidence of facilitated cue encoding (e.g., Logan \& Bundesen, 2003; Schneider \& Logan, 2005, 2007).

A final prediction concerns task transition effects. A frequent observation in task-switching studies is that performance is faster for task repetitions compared with task switches (for a review, see Monsell, 2003). However, recent studies have demonstrated that these task-repetition benefits (often called task-switch costs) can be absent or reversed when subjects perform tasks organized in a hierarchical representation (Lien \& Ruthruff, 2004; Schneider \& Logan, 2006). One way of interpreting this finding is that task activation is linked to specific units in the hierarchical represen- 


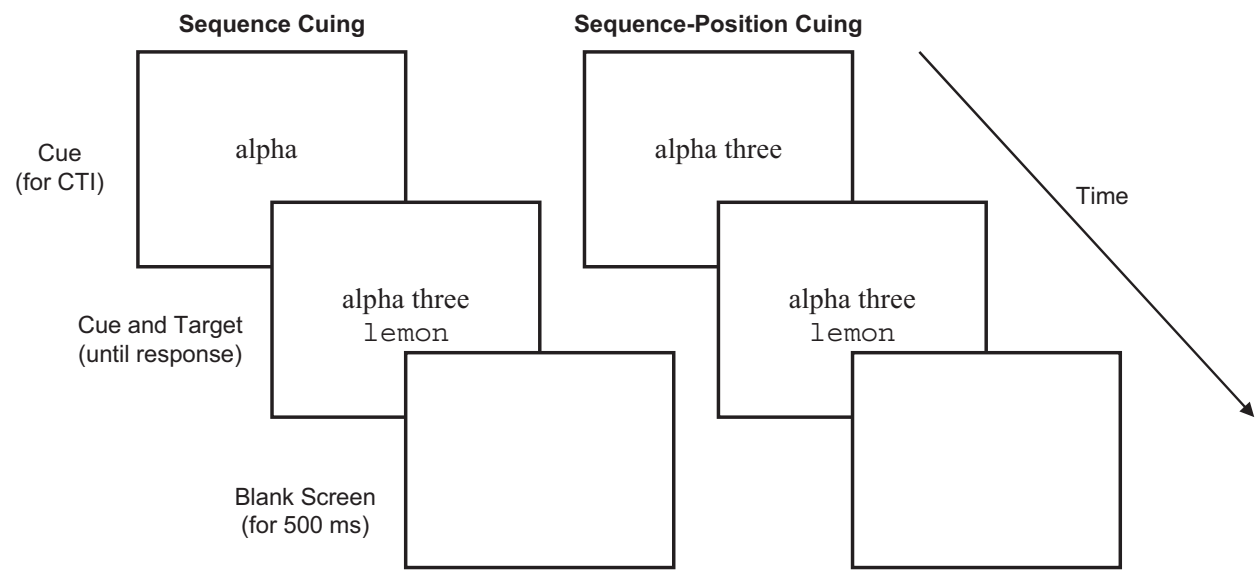

Figure 2. Schematic of events during each trial for the sequence and sequence-position cuing conditions. CTI $=$ cue-target interval.

tation and there is no benefit to accessing different units during various sequence transitions, even if those units are associated with the same task. It is also possible that sequence-level processing (e.g., switching sequences) disrupts the elements of task-level processing that would otherwise produce task-repetition benefits, an outcome that Schneider and Logan (2006) interpreted as evidence of hierarchical control. Regardless of the interpretation, previous research leads to the prediction of little or no taskrepetition benefit, though we note that this prediction rests on the assumption that previous findings can be generalized to the sequence-position cuing procedure.

Non-hierarchical representation. If information is retrieved from a non-hierarchical representation, then a different pattern of results would be expected. One might expect little or no sequencerepetition benefit because knowledge units are not organized into higher level sequences. If a unit can only be accessed with the combination of the sequence and position parts of the cue (by analogy, think of needing two keys to open a lock), then a benefit of accessing the same knowledge unit would only occur for situations involving sequence repetitions and position repetitions, yielding a small sequence-repetition benefit (because only $25 \%$ of sequence repetitions for the task sequences in Figure 1B would be associated with a benefit). A less restricted form of retrieval would allow for part of a cue to access several units in parallel (e.g., the cue alpha could access all four alpha units in Figure 1B). In this case, a larger sequence-repetition benefit is possible, and it would have similar temporal dynamics with respect to CTI as noted above. Thus, the mere presence of a sequence-repetition benefit is not unequivocal evidence of a hierarchical representation.

Position-repetition benefits would be expected regardless of the sequence transition. This prediction differs from that for the hierarchical representation, wherein the benefit should be restricted to sequence repetitions. For the non-hierarchical representation, if part of a cue can access several units in parallel (e.g., the cue three could access both three units in Figure 1B), then a positionrepetition benefit should occur even in the case of sequence switches (e.g., beta three $\rightarrow$ alpha three).

Form transitions would modulate sequence- and positionrepetition benefits in the same manner as for a hierarchical repre- sentation. Evidence in support of this prediction comes from previous studies of cue-repetition benefits in task switching in which tasks were not represented in a structured manner (e.g., Logan \& Bundesen, 2003; Schneider \& Logan, 2005, 2007).

Finally, task-repetition benefits would be expected regardless of the sequence transition. This prediction differs from that for the hierarchical representation, wherein there should be little or no task-repetition benefits. For the non-hierarchical representation, sequence transitions should have no effect because task units are not linked to higher level sequence units- the latter do not exist (see Figure 1B). As a corollary, task-level processing that typically produces task-repetition benefits would not be disrupted by sequence-level processing because the latter would not occur. In this situation, given that sequence-position cues are functionally equivalent to arbitrary task cues, there should be large, robust task-repetition benefits, as observed in many task-switching studies (e.g., Logan \& Bundesen, 2004; Mayr \& Kliegl, 2003; for a summary from several experiments, see Logan, Schneider, \& Bundesen, 2007).

In summary, several effects are expected, and there are at least two divergent predictions associated with hierarchical and nonhierarchical representations. A hierarchical representation should yield a position-repetition benefit for sequence repetitions, but little or no benefit for sequence switches, as well as small or null task-repetition benefits. A non-hierarchical representation should yield position-repetition benefits regardless of the sequence transition, as well as large task-repetition benefits in all conditions. A critical point to bear in mind is that position transition and task transition are partially confounded, making it necessary to consider whether one type of transition effect may reflect the other. (We show below that task-repetition benefits in the sequence-position cuing procedure largely reflect position-repetition benefits.)

To foreshadow the results, we found that the data were consistent with a hierarchical representation and inconsistent with a non-hierarchical representation. Following the presentation of the results, we introduce a mathematical model that was developed to check the validity of the verbal arguments underlying the above predictions and to better understand the nature of retrieval from a hierarchical representation. We show that with a relatively small 
set of assumptions and equations, the model is able to capture the major effects in the data, providing a concrete demonstration of how non-isomorphic behavior in the context of the sequenceposition cuing procedure can be understood in terms of retrieval from a hierarchical plan.

\section{Method}

\section{Subjects}

Sixty-four students from Vanderbilt University completed the experiment for course credit or $\$ 12$. Thirty-two students were assigned to each cuing condition.

\section{Apparatus}

The experiment was conducted with E-Prime software (Psychology Software Tools, Pittsburgh, PA) running on computers that displayed stimuli on monitors and registered responses from keyboards.

\section{Tasks and Targets}

The tasks were origin (living or nonliving) and size (small or large, relative to a basketball) judgments. The targets were 64 words drawn from a larger set used by Arrington and Logan (2004), with 16 words for each combination of the origin and size categories. Mean word length was 6.2 letters, and mean word frequency was 7.4 per million; separate 2 (origin) $\times 2$ (size) analyses of variance (ANOVAs) on each variable revealed no significant effects (all $F s<1$ ). All targets were displayed in white 12-point Courier New font on a black background and viewed at a distance of about $60 \mathrm{~cm}$.

\section{Sequences}

Each subject was assigned two task sequences, labeled alpha and beta (as words and as the corresponding Greek letters $\alpha$ and $\beta)$. There were four possible pairs of sequences: OOSS-OSSO, OOSS-SOOS, SSOO-OSSO, and SSOO-SOOS, where O and S denote the origin and size tasks, respectively, and sequences are separated by hyphens. The designation of each sequence as alpha or beta was counterbalanced across subjects and all pairs of sequences were used equally often. All sequences are matched for task and task transition frequency (across iterations of a sequence). During the instructions, each sequence was displayed onscreen as comma-delimited uppercase words (e.g., ORIGIN, ORIGIN, SIZE, SIZE for the OOSS sequence).

\section{Procedure}

Instructions were presented onscreen and explained by the experimenter. The experiment was divided into two phases. In the first phase, subjects completed practice blocks of trials to facilitate memorization of each sequence, as in Schneider and Logan (2006). Each practice block was preceded by a display that reminded subjects about the sequences. The next display consisted of a word (alpha or beta) or a Greek letter ( $\alpha$ or $\beta$ ) that was randomly selected and presented in the center of the screen. The word or letter was a prompt indicating the sequence that was to be per- formed repeatedly across trials during that block. When ready, subjects initiated the block, at which point the prompt disappeared.

Each trial in a practice block began with the onset of a randomly selected target in the center of the screen. The target remained onscreen until the subject pressed either the $Z$ key with the left index finger or the forward slash (/) key with the right index finger. Response categories for the same task were assigned to different response keys, and all possible response-key mappings were counterbalanced across subjects. Text indicating the response-key mappings appeared in the bottom corners of the screen. After a response, the target was erased, and the next trial commenced after a response-cue interval of $500 \mathrm{~ms}$. Subjects had to rely on their memory of the sequence and keep track of the relevant serial position across trials, restarting the sequence every four trials. Each practice block included 16 trials, enabling four iterations of the sequence. Subjects completed eight blocks (four per sequence, half prompted by a word and half prompted by a letter) separated by rest periods. Each of the 64 targets appeared once every four blocks. After the practice blocks, the experimenter was summoned to give further instructions.

In the second phase, subjects received instructions about sequence-position cues. Each cue had two parts: a sequence part and a position part. The sequence part was a word or a letter indicating a sequence (e.g., alpha or $\alpha$ ). The position part was a word or a digit indicating a serial position in that sequence (e.g., three or 3). When combined, these parts form a sequence-position cue that can be used to determine the task to perform on a trial. For example, consider a subject who has OOSS as the alpha sequence and SOOS as the beta sequence. The cue alpha three (or $\alpha 3$ ) indicates the third task in the alpha sequence-the size task; the cue beta two (or $\beta$ 2) indicates the second task in the beta sequence-the origin task (see Figure 1). These examples were used in the instructions to explain the cues. The experimenter indicated that all possible sequence-position cues would be presented during the experiment and that they would appear in either word form (e.g., alpha three) or letter-digit form (e.g., $\alpha 3$ ). Subjects had to use the cue presented on each trial to determine which task to perform on the target, giving their best effort to respond quickly and accurately. The tasks, targets, response-key mappings, and sequences remained the same as in the practice blocks. The block procedure was modified by omitting the sequence reminder and prompt displays preceding each block. The trial procedure was modified by displaying a cue in white 12-point bold Times New Roman font $18 \mathrm{~mm}$ above the target.

The onsets of the sequence and position parts of the cue were manipulated between subjects, as shown in Figure 2. In the sequence cuing condition, only the sequence part was presented at the start of each trial. After a variable CTI $(0 ; 300 ; 700 ; 1,100$; or $1,500 \mathrm{~ms})$, the position part appeared $30 \mathrm{~mm}$ to the right of the sequence part, simultaneously with the target (see Figure 2). The cue and the target remained onscreen until a response was registered, at which time the screen went blank and the next trial commenced after $500 \mathrm{~ms}$. In the sequence-position cuing condition, both parts of the cue were presented simultaneously at the start of each trial (see Figure 2). After the CTI, the target appeared, and the trial proceeded as in the sequence cuing condition. Subjects were told that one (sequence cuing condition) or both (sequence-position cuing condition) parts of the cue would appear in advance of the target and they were instructed to use the 
advance information to prepare for subsequent performance. There were 80 trials per block, representing one replication of an 8 (cue) $\times 2$ (cue form) $\times 5$ (CTI) block design, with the specific combination of these variables randomly selected on each trial. In other words, sequence, position, form, and (by extension) task transitions occurred in random order, so the series of tasks performed across trials did not consistently correspond to the serial order of the tasks in either sequence. Subjects completed eight blocks (separated by rest periods) for a total of 640 trials.

\section{Results and Discussion}

Practice blocks, the first trial of each block, and trials with RT exceeding 5,000 ms (3.9\% of trials) were excluded from all analyses. Trials with incorrect responses were excluded from the RT analysis. Sequence, task, form, and position transitions were coded for every trial. As noted earlier, task transition is partially confounded with position transition; therefore, we conducted separate analyses in which either task transition or position transition was a variable in the ANOVA design.

\section{Time Course Analysis}

To analyze the time course of performance, we calculated mean RT and error rate (ER) for each subject in each cuing condition for every combination of four variables: sequence transition, task transition, form transition, and CTI. These data are provided in Appendix A and were submitted to separate 2 (cuing condition) $\times$ 2 (sequence transition) $\times 2$ (task transition) $\times 2$ (form transition) $\times 5(\mathrm{CTI})$ mixed-measures ANOVAs, with cuing condition as a between-subjects variable and all other variables as withinsubject variables. The ANOVA results are summarized in Appendix B. For the RT data, all main effects were significant, but each variable was involved in at least one significant interaction (see Appendix B); therefore, we focus on the interactions.
CTI effects. Mean RT is plotted as a function of CTI, sequence transition, and form transition for each cuing condition in Figure 3. RT decreased as CTI increased in each cuing condition, but the time-course function was much steeper-and RT at the longest CTI was shorter-for the sequence-position cuing condition compared with the sequence cuing condition (reflecting the significant interaction between cuing condition and CTI). These findings are consistent with the idea that more information was retrieved during the CTI in the sequence-position cuing condition than in the sequence cuing condition. Sequence, position, and task information could be retrieved during the CTI in the sequence-position cuing condition, whereas only sequence information could be retrieved during the $\mathrm{CTI}$ in the sequence cuing condition (position and task information had to be retrieved upon target onset). As the amount of information retrieved during the CTI increases, the amount of information to be retrieved upon target onset decreases, facilitating performance.

Sequence transition effects. The significant interaction between sequence transition and CTI is also illustrated in Figure 3. Sequence repetitions were faster than sequence switches, replicating previous findings (e.g., Lien \& Ruthruff, 2004; Luria \& Meiran, 2003; Schneider \& Logan, 2006). Moreover, these sequencerepetition benefits decreased as CTI increased. As CTI increased from 0 to $1,500 \mathrm{~ms}$, the sequence-repetition benefit decreased from 357 to $210 \mathrm{~ms}$ in the sequence cuing condition and from 315 to 216 $\mathrm{ms}$ in the sequence-position cuing condition; these declines were not significantly different (see Appendix B). These findings are consistent with the idea that there is a benefit associated with retrieving information from the same knowledge unit across trials. Furthermore, the decrease in the sequence-repetition benefit across CTI indicates that retrieval of sequence information occurred in advance of target onset in both cuing conditions. Although these results are consistent with retrieval from a hierarchical representation, they do not exclude the possibility of parallel retrieval from
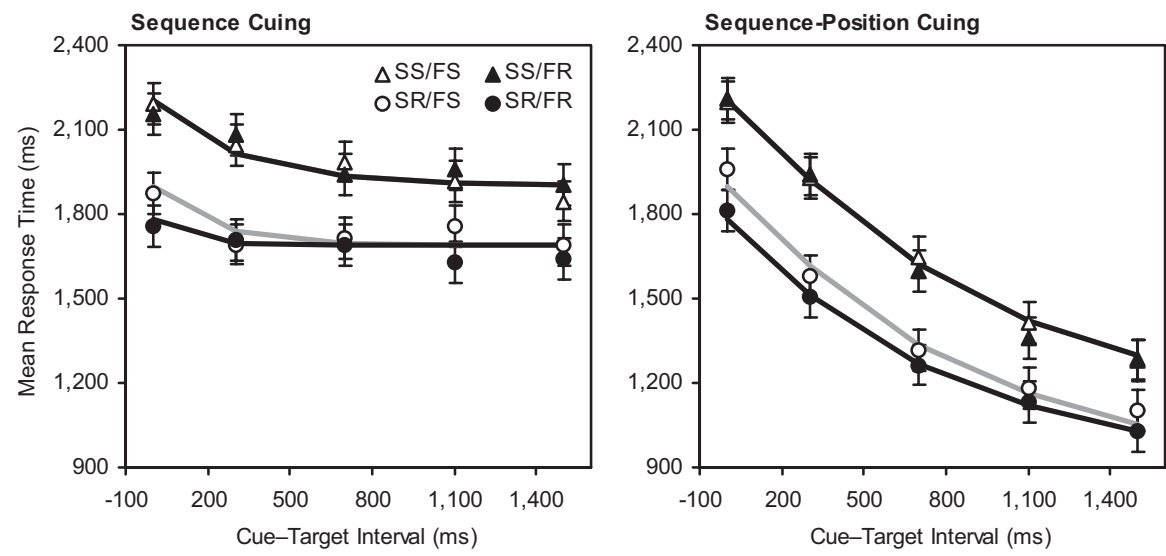

Figure 3. Mean response time as a function of cue-target interval, sequence transition (SS = sequence switch, $\mathrm{SR}=$ sequence repetition), and form transition $(\mathrm{FS}=$ form switch, $\mathrm{FR}=$ form repetition) for the sequence and sequence-position cuing conditions. Points represent observed data. Black and gray lines represent model predictions for form repetitions and form switches, respectively; for sequence switches, the lines completely overlap. Error bars represent $95 \%$ confidence intervals calculated using the error term associated with the Cue Type $\times$ Sequence Transition $\times$ Form Transition $\times$ Cue-Target Interval interaction from the relevant analysis of variance on the data. 
a non-hierarchical representation. However, the relatively large sequence-repetition benefits and their change with CTI are evidence against the restricted form of retrieval from a nonhierarchical representation, whereby a knowledge unit can only be accessed when both parts of the cue are available.

Form transition effects. If the sequence-repetition benefits arise from accessing the same knowledge unit across trials, then there should be an additional benefit of using the same cue to access the same knowledge unit. The nature of the significant interaction between sequence transition and form transition supports this idea. When repeating sequences, form repetitions (e.g., word form $\rightarrow$ word form) were $70 \mathrm{~ms}$ faster than form switches (e.g., letter-digit form $\rightarrow$ word form), whereas when switching sequences, form repetitions were only $2 \mathrm{~ms}$ faster than form switches (see Figure 3). Note that form repetitions are sequencecue repetitions when repeating sequences (e.g., alpha $\rightarrow$ alpha), but not when switching sequences (e.g., beta $\rightarrow$ alpha), indicating that the form-repetition benefit is a cue-repetition benefit (Logan \& Bundesen, 2003; Schneider \& Logan, 2005, 2007).

Given that sequence-cue encoding can occur during the CTI in each cuing condition, the contribution of the cue-repetition benefit to RT should decrease with CTI. The interaction between form transition and CTI, as well as the four-way interaction between cuing condition, sequence transition, form transition, and CTI, were marginally significant (see Appendix B). The four-way interaction reflects different time courses for the cue-repetition benefits associated with sequence repetitions in each cuing condition. In the sequence cuing condition, the cue-repetition benefit disappears by the CTI of $300 \mathrm{~ms}$ (except for a brief reappearance at the CTI of 1,100 ms), whereas in the sequence-position cuing condition, it decreases more gradually across CTI (see Figure 3 ). The time courses of the cue-repetition benefits are generally consistent with those observed in previous work (e.g., Logan \& Bundesen, 2003; Schneider \& Logan, 2005).

Task transition effects. By accessing the appropriate knowledge unit(s), the relevant task can be identified and performed, resulting in either a task repetition or a task switch from the preceding trial. Recall that there are divergent predictions concerning task transition effects based on retrieval from hierarchical and non-hierarchical representations. A hierarchical representation should yield small or null taskrepetition benefits, whereas a non-hierarchical representation should yield large task-repetition benefits in all conditions.

In the data, task repetitions were faster than task switches, but these task-repetition benefits varied with sequence transition (as indicated by the significant interaction between sequence transition and task transition). For sequence repetitions, there were task-repetition benefits of 168 and $181 \mathrm{~ms}$ in the sequence and sequence-position cuing conditions, respectively, but for sequence switches, there were (nonsignificant) task-repetition costs of 17 and $11 \mathrm{~ms}$ in the sequence and sequence-position cuing conditions, respectively. The latter effects are strikingly similar to the nonsignificant task-repetition cost of $16 \mathrm{~ms}$ for sequence switches observed by Schneider and Logan (2006, Experiment 4) using a different procedure with different tasks.

At first glance, these findings do not seem to provide conclusive support for retrieval from either a hierarchical or a nonhierarchical representation. The null task-repetition benefit for sequence switches is consistent with a hierarchical representation but is highly problematic for a non-hierarchical representation, for which task-repetition benefits should occur independently of sequence switching because tasks are not organized at a sequence level (see Figure 1B). In contrast, the large task-repetition benefit for sequence repetitions is consistent with a non-hierarchical representation but does not seem to fit with a hierarchical representation- under the assumption that previous findings (e.g., Lien \& Ruthruff, 2004; Schneider \& Logan, 2006) generalize to the sequence-position cuing procedure. However, recall that task transition is partially confounded with position transition, specifically for sequence repetitions, for which position repetitions are always task repetitions. It is possible that the observed task-repetition benefit may actually be a position-repetition benefit. For this reason, we defer making strong conclusions about this aspect of the task transition data until position transitions are analyzed in the next section.

There were four remaining significant interactions in the time course analysis of the RT data (see Appendix B). The interaction between task transition and CTI is qualified by a higher order interaction involving sequence transition. For sequence switches, the task-repetition benefit did not vary systematically across CTI, vacillating between -59 and $10 \mathrm{~ms}$. For sequence repetitions, the task-repetition benefit decreased from 245 to $80 \mathrm{~ms}$ as the CTI increased from 0 to $1,500 \mathrm{~ms}$. The interaction between cuing condition, task transition, and form transition is qualified by a higher order interaction involving sequence transition that was not interpretable.

For the ER data, only one main effect and three interactions were significant (see Appendix B). Task repetitions were more accurate than task switches, but the main effect of task transition is qualified by a significant interaction with cuing condition, reflecting a higher task-repetition benefit in the sequence cuing condition $(1.3 \%)$ than in the sequence-position cuing condition $(0.5 \%)$. There was also a significant interaction between cuing condition and sequence transition, reflecting a sequence-repetition benefit of $0.2 \%$ in the sequence-position cuing condition, but a sequence-repetition cost of $0.7 \%$ in the sequence cuing condition. The latter effect is suggestive of a speed-accuracy tradeoff in the sequence cuing condition, but a follow-up analysis provides some evidence against this possibility. ${ }^{1}$ The only remaining significant effect was an interaction between sequence transition and CTI, but the change in the sequence-repetition benefit across CTI did not conform to any interpretable pattern.

\section{Position Transition Analysis}

To analyze position transition effects and to ascertain whether the task-repetition benefit we observed for sequence repetitions in

\footnotetext{
${ }^{1}$ If subjects sacrificed accuracy for speed in the sequence cuing condition, then one might expect error responses to be faster than correct responses. Mean RTs were calculated for error and correct responses for each sequence transition in the sequence cuing condition, collapsing over all other variables and excluding 2 subjects who did not make any errors for either sequence switches or sequence repetitions. A 2 (sequence transition) $\times 2$ (response type) repeated-measures ANOVA revealed a significant main effect of sequence transition (which merely replicates the earlier analysis that excluded error trials), but more importantly, the main effect of response type and the interaction between sequence transition and response type were nonsignificant (both $F \mathrm{~s}<1$ ), mitigating the possibility of a speed-accuracy tradeoff.
} 
the preceding analysis may actually be a position-repetition benefit, we first conducted a 2 (cuing condition) $\times 2$ (sequence transition) $\times 2$ (position transition) $\times 2$ (form transition) mixedmeasures ANOVA, collapsing across task transition and CTI to permit an adequate number of observations for analysis. Mean RTs contributing to the analysis are presented in Figure 4. To avoid redundancy, we report only effects associated with position transition.

There was a significant main effect of position transition, $F(1$, $62)=83.1, M S E=30,780, p<.001, \eta_{p}{ }^{2}=.57$, such that position repetitions were $141 \mathrm{~ms}$ faster than position switches. This position-repetition benefit was qualified by a significant interaction with sequence transition, $F(1,62)=61.4, M S E=21,715, p<$ $.001, \eta_{p}{ }^{2}=.50$, and a marginally significant interaction with form transition, $F(1,62)=3.5, M S E=15,574, p=.06, \eta_{p}{ }^{2}=.05$, as well as a marginally significant interaction with both sequence transition and form transition, $F(1,62)=3.3, M S E=12,145, p=$ $.07, \eta_{p}{ }^{2}=.05$. The three-way interaction reflects different patterns of data for sequence switches and sequence repetitions. For sequence switches, there is little or no effect of position transition or form transition (see Figure 4). For sequence repetitions, position repetitions were faster than position switches, and form repetitions were faster than form switches, though this latter effect was predominantly for position repetitions (see Figure 4).

These findings are consistent with the idea that there is a benefit associated with accessing the same knowledge unit across trials, especially when the same cue is used to access that unit. More importantly, they address the divergent predictions for retrieval from hierarchical and non-hierarchical representations. Recall that a hierarchical representation should yield a position-repetition benefit for sequence repetitions but not for sequence switches, whereas a non-hierarchical representation should yield a positionrepetition benefit regardless of the sequence transition. The data are consistent with the hierarchical representation and inconsistent with the non-hierarchical representation.

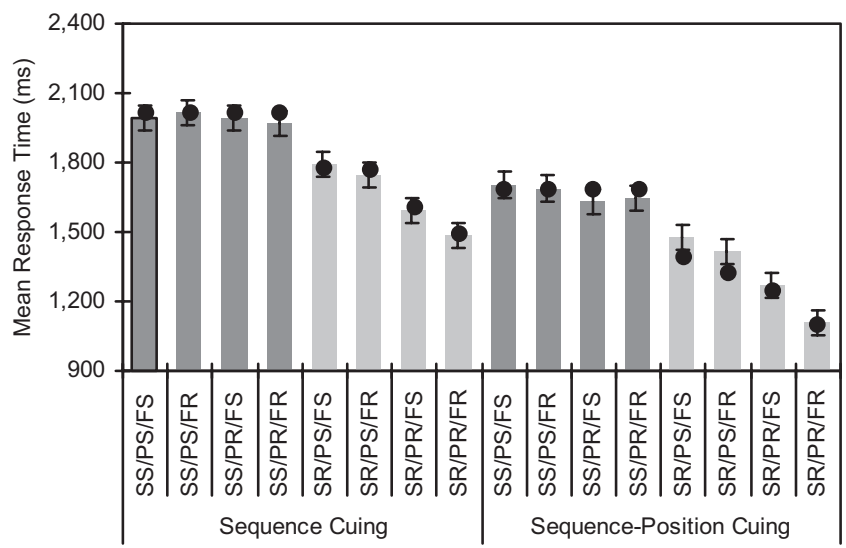

Figure 4. Mean response time as a function of sequence transition ( $\mathrm{SS}=$ sequence switch, $\mathrm{SR}=$ sequence repetition), position transition (PS = position switch, $\mathrm{PR}=$ position repetition), and form transition $(\mathrm{FS}=$ form switch, FR = form repetition) for the sequence and sequence-position cuing conditions. Bars represent observed data. Points represent model predictions. Error bars represent $95 \%$ confidence intervals calculated using the error term associated with the Cue Type $\times$ Sequence Transition $\times$ Position Transition $\times$ Form Transition interaction from the relevant analysis of variance on the data.
The same ANOVA on the ER data revealed a significant main effect of position transition, $F(1,62)=5.1, M S E=6, p<.05$, $\eta_{p}{ }^{2}=.08$, such that position repetitions were $0.4 \%$ more accurate than position switches. This position-repetition benefit was qualified by a significant interaction with sequence transition, $F(1$, 62) $=11.9, M S E=5, p<.005, \eta_{p}{ }^{2}=.16$, and a significant interaction with both cuing condition and sequence transition, $F(1$, 62) $=4.4, M S E=5, p<.05, \eta_{p}{ }^{2}=.07$. The interactions reflect the fact that position repetitions were $1.1 \%$ more accurate than position switches for sequence repetitions (an effect that was greater in the sequence cuing condition [1.9\%] than in the sequence-position cuing condition $[0.4 \%])$, but there was no such difference $(-0.2 \%)$ for sequence switches. The finding that position-repetition benefits in the ER data were restricted to sequence repetitions is consistent with the pattern in the RT data.

To determine whether the observed task-repetition benefit from the time course analysis is actually a position-repetition benefit, we recomputed the earlier 2 (cuing condition) $\times 2$ (sequence transition) $\times 2$ (task transition) $\times 2$ (form transition) $\times 5(\mathrm{CTI})$ mixed-measures ANOVA on the RT data, excluding trials with position repetitions. In contrast to the original analysis (see Appendix B), the reanalysis yielded no main effect of task transition ( $p=.13$; mean task-repetition benefit $=22 \mathrm{~ms})$; no interaction between cuing condition, task transition, and form transition ( $p=$ .13 ); no interaction between task transition and CTI ( $p=.74)$; and no interaction between sequence transition, task transition, and CTI $(p=.08)$. None of the non-task transition effects in the original analysis were affected, except for the main effect of form transition, which became marginally significant ( $p=.051)$. In the same ANOVA on the ER data, there was no longer an interaction between cuing condition and task transition $(p=.16)$.

These results indicate that the task-repetition benefits in the data are primarily position-repetition benefits. The lack of robust taskrepetition benefits across conditions is consistent with a hierarchical representation but inconsistent with a non-hierarchical representation. When the task transition effects from the recomputed time course analysis are considered in conjunction with the position transition effects, the data provide strong support for retrieval from a hierarchical representation.

\section{Modeling}

Modeling has played an important role in the debate about whether routine sequential action involves hierarchical or nonhierarchical representations (Botvinick \& Plaut, 2004; Cooper \& Shallice, 2000), in part because it has allowed researchers to demonstrate that a given type of representation is sufficient to account for performance. In the present study, to say that our data are consistent with retrieval from a hierarchical representation is one thing; to show explicitly that this is the case is far more compelling than verbal arguments alone. Whereas our arguments may be valid in principle, they may not necessarily be valid in practice. For this reason, we developed a mathematical model of retrieval in the context of the sequence-position cuing procedure. In this section, we spell out the core assumptions of the model, explain how these assumptions are formally implemented, then show that the model accounts for the complex pattern of results from our experiment. 


\section{Assumptions}

The model is based on the assumptions that retrieval from a hierarchical representation is time-consuming, susceptible to priming, cue-dependent, structurally constrained, and token-specific. We are by no means the first to invoke such assumptions. Indeed, each of these assumptions has some precedence in the history of cognitive psychology, providing a strong theoretical foundation for the model.

Retrieval is time-consuming in that it takes time to retrieve information from a knowledge unit at a given level of the hierarchical representation in memory. The notion that retrieval takes time is a common assumption in theorizing about memory retrieval (e.g., Collins \& Quillian, 1969; Ratcliff, 1978), especially in accounts of serial retrieval from hierarchical representations (Anderson et al., 1998; Anderson \& Matessa, 1997; Povel \& Collard, 1982; Rosenbaum et al., 1983). The end product of retrieval is information from an activated knowledge unit, but in the absence of supporting input, we assume that activation decays over time. However, residual activation may persist across trials, and retrieval is susceptible to priming in that this residual activation facilitates re-retrieval of information. The result would be a repetition benefit when the same knowledge unit is accessed across trials. We assume that a similar type of repetition priming can occur during cue encoding, with residual activation of a cue representation in memory facilitating re-encoding of that cue (Logan \& Bundesen, 2003; Schneider \& Logan, 2005).

Retrieval is cue-dependent in that a knowledge unit can only be activated when an associated cue is presented. The notion of cue-dependent retrieval is a common assumption in theorizing about recognition and recall (e.g., Gillund \& Shiffrin, 1984; Hintzman, 1986; Tulving \& Thomson, 1973) and in the sequenceposition cuing procedure, we assume that retrieval is based on external (rather than internal) cues. However, cue-based retrieval is structurally constrained in that low-level information can only be retrieved if high-level information has already been accessed. This assumption is consistent with theorizing about serial retrieval (e.g., Anderson et al., 1998; Anderson \& Matessa, 1997; Collard \& Povel, 1982; Rosenbaum et al., 1983; see also Johnson, 1970), and it follows that retrieval occurs from high to low levels in the hierarchical representation, or more specifically, from the sequence level to the position level (possibly mirroring how the representation was constructed; see Dixon, 1982, 1987). Although we assume that retrieval of information from different levels occurs in a serial, discrete manner, we do not exclude the possibility of parallel or cascaded retrieval (McClelland, 1979; see also Schneider \& Logan, 2006, p. 628). Finally, retrieval is tokenspecific in that if the same information is contained in low-level knowledge units nested under different high-level knowledge units (e.g., the origin two units in Figure 1A), activating one of these low-level units does not entail activation of the other units. Thus, we are making a type-token distinction, whereby such units may be of the same type, but they are represented and retrieved as unique tokens in the hierarchical representation.

The first three assumptions (time-consuming retrieval, susceptibility to priming, and cue dependency) are not uniquely associated with retrieval from a hierarchical representation; these same assumptions would be consistent with a non-hierarchical representation. However, the last two assumptions (structural constraint and token specificity) are strongly associated with hierarchical representations, and they are largely inconsistent with nonhierarchical representations. With respect to structural constraint, a non-hierarchical representation would not have retrieval from high to low levels because there are no differing representational levels (see Figure 1B). With respect to token specificity, knowledge units with the same information could be represented as separate tokens in a non-hierarchical representation, but retrieval would not necessarily be restricted to a specific token if a cue is associated with multiple tokens.

\section{Model Formulation}

The assumptions of time-consuming retrieval, susceptibility to priming, and cue dependency are built into the architecture of the model, whereas the assumptions of structural constraint and token specificity are primarily associated with the application of the model to data.

Parameters. The model is formulated as a set of equations based on two types of parameters: $\mu$ and $R T_{\text {Base. The } \mu \text { parameter }}$ represents mean retrieval time, which includes the time to encode a cue and to retrieve the information associated with the cue (i.e., to activate the relevant knowledge unit). We assume there are separate $\mu$ parameters for retrieval of sequence information $\left(\mu_{S}\right)$ and retrieval of position information $\left(\mu_{\mathrm{P}}\right)$. Given that the sequence information retrieved on a sequence-repetition trial is the same as that on the preceding trial, retrieval time for sequence repetitions $\left(\mu_{\mathrm{SR}}\right)$ is expected to be faster than that for sequence switches $\left(\mu_{\mathrm{SS}}\right)$ due to residual activation of the relevant sequence knowledge unit, leading to a sequence-repetition benefit, with $\mu_{\mathrm{SR}}<\mu_{\mathrm{SS}}$.

Given that $\mu$ encompasses cue-encoding time, retrieval time for sequence repetitions involving form repetitions (sequence-cue repetitions, e.g., alpha $\rightarrow$ alpha) is expected to be faster than that for sequence repetitions involving form switches (e.g., $\alpha \rightarrow$ alpha) due to residual activation of the cue representation used to access that unit. For this reason, there are separate $\mu$ parameters for sequence-cue repetitions $\left(\mu_{\mathrm{SCR}}\right)$ and sequence repetitions involving form switches $\left(\mu_{\mathrm{SR}}\right)$, leading to a cue-repetition benefit, with $\mu_{\mathrm{SCR}}<\mu_{\mathrm{SR}}$. We do not distinguish between different types of sequence switches in the model because even in the case of form repetitions, the cue representation on the current trial will still be different from that on the preceding trial (e.g., alpha $\rightarrow$ beta), so there would be no cue-encoding benefit. Thus, there are only three $\mu_{\mathrm{S}}$ parameters in the model, with $\mu_{\mathrm{SCR}}<\mu_{\mathrm{SR}}<\mu_{\mathrm{SS}}$.

In our application of the model to the time course data in Figure 3, we assume only one parameter associated with position retrieval $\left(\mu_{\mathrm{P}}\right)$. For modeling the position transition data in Figure 4 , we assume there is a set of $\mu_{P}$ parameters that mirrors the set of $\mu_{\mathrm{S}}$ parameters: $\mu_{\mathrm{PCR}}$ for position-cue repetitions (e.g., three $\rightarrow$ three), $\mu_{\mathrm{PR}}$ for position repetitions (e.g., $3 \rightarrow$ three), and $\mu_{\mathrm{PS}}$ for position switches (e.g., one $\rightarrow$ three), leading to cue- and positionrepetition benefits, with $\mu_{\mathrm{PCR}}<\mu_{\mathrm{PR}}<\mu_{\mathrm{PS}}$. However, a critical feature of the model is that the application of these parameters is structurally constrained, such that a condition is only treated as a position repetition in the model if the same position unit in the hierarchical representation is accessed across trials. In other words, a transition such as alpha three $\rightarrow \alpha 3$ is treated as a position repetition in the model, but a transition such as alpha three $\rightarrow$ beta three is treated as a position switch—even though it was a nominal 
position repetition in the data analysis-because different position units are accessed (see Figure 1A). Although there is the possibility of partial cue-repetition benefits for position repetitions on sequence-switch trials, such effects were minimal in the data (see Figure 4), so we did not introduce additional $\mu_{\mathrm{P}}$ parameters to model them.

The $R T_{\text {Base }}$ parameter represents residual processing time, which encompasses the time associated with all processes beyond sequence and position retrieval, such as those involved in task performance proper. As with the $\mu$ parameters, we assume there are separate $R T_{\text {Base }}$ parameters for sequence repetitions and sequence switches $R T_{\text {Base-SR }}$ and $R T_{\text {Base-sS }}$, respectively), reflecting the idea that a repeated sequence unit not only may be accessed faster but also may be more active during a sequence-repetition trial, thereby facilitating performance (hence, $R T_{\text {Base-SR }}<$ $\left.R T_{\text {Base-ss }}\right)$. We do not assume a separate $R T_{\text {Base }}$ parameter for sequence-cue repetitions because we interpret cue-repetition benefits to be primarily perceptual effects associated with cue encoding - a process subsumed within the $\mu$ parameter.

Equations. For both cuing conditions, we assume that sequence information can be retrieved during the CTI because the sequence part of the cue is presented at the start of each trial. However, it is only in the sequence-position cuing condition that position information can also be retrieved during the CTI. In the sequence cuing condition, position information can only be retrieved upon target onset. These differences in retrieval across cuing conditions are captured by the following equations, which are based on equations developed by Logan and Bundesen (2003) to model cue-encoding and set-switching processes in explicitly cued task-switching performance (though they represent different processes in our model).

For the sequence cuing condition (see Logan \& Bundesen, 2003, p. 577, Equation 1):

$$
R T=R T_{\text {Base }}+\mu_{\mathrm{s}} \cdot \exp \left(-\mathrm{CTI} / \mu_{\mathrm{s}}\right)+\mu_{\mathrm{P}} .
$$

In Equation 1, retrieval of sequence information-but not position information-occurs during the CTI because only the sequence part of the cue is available prior to target onset. We assume that sequence retrieval time is exponentially distributed, such that its contribution to RT decreases as CTI increases, producing a monotonically decreasing time-course function. As a corollary, any differences in sequence retrieval time decrease as CTI increases; therefore, cue- and sequence-repetition benefits get smaller. Although we also assume that position retrieval time is exponentially distributed, retrieval can only begin upon presentation of the position part of the cue, which occurs after the CTI in the sequence cuing condition. The same constraint applies to task performance proper. For this reason, position retrieval time and residual processing time are additive terms in Equation 1.

For the sequence-position cuing condition (see Logan \& Bundesen, 2003, p. 577, Equation 2):

$$
\begin{gathered}
R T=R T_{\text {Base }}+\exp \left(-\mathrm{CTI} / \mu_{\mathrm{S}}\right) \cdot\left(\mu_{\mathrm{S}}+\mu_{\mathrm{P}}\right) \\
+\frac{1 / \mu_{\mathrm{S}}}{1 / \mu_{\mathrm{S}}-1 / \mu_{\mathrm{P}}} \cdot\left[\exp \left(-\mathrm{CTI} / \mu_{\mathrm{P}}\right)-\exp \left(-\mathrm{CTI} / \mu_{\mathrm{S}}\right)\right] \cdot \mu_{\mathrm{P}}
\end{gathered}
$$

In Equation 2, retrieval of both sequence and position information occurs during the CTI because both parts of the cue are available prior to target onset. Sequence retrieval and position retrieval are serial processes (reflecting the structural constraint assumption) with exponentially distributed finishing times and, as in Equation 1, the contribution of retrieval time to RT decreases as CTI increases. However, given that more information is retrieved during the CTI, Equation 2 yields a steeper time-course function than does Equation 1.

\section{Model Fits}

We first report a fit of the model to the time course data in Figure 3. We constrained the model such that the same values for the six free parameters $\left(\mu_{\mathrm{SCR}}, \mu_{\mathrm{SR}}, \mu_{\mathrm{SS}}, \mu_{\mathrm{P}}, R T_{\mathrm{Base}-\mathrm{SR}}\right.$, and $R T_{\text {Base-SS }}$ ) were used to fit both the sequence and sequenceposition cuing data simultaneously (a total of 40 data points). This is a strong constraint because it is based on the assumption that the only difference between cuing conditions is whether position retrieval occurs after or during the CTI (Equation 1 vs. Equation 2). The specific parameters used in Equations 1 and 2 to model each combination of sequence transition and form transition are provided in Table 1. We fit the model to the data using the Solver function in Microsoft Excel to minimize the root mean-squared deviation (RMSD) between observed and predicted RTs (the product-moment correlation, $r$, was also computed).

The model had a good fit to the data, with RMSD $=35 \mathrm{~ms}$ and $r=.994$. The model predictions are plotted as lines in Figure 3 and the best-fitting parameter values are provided in Table 2 (note that the model predictions in Figure 3 can be generated by plugging the values from Table 2 into Equations 1 and 2 as per the specifications in Table 1). All of the model predictions fell within the $95 \%$ confidence intervals plotted around the data points in Figure 3. Moreover, the model captured the major effects from the time course analysis. The model produced sequence-repetition benefits because $\mu_{\mathrm{SR}}<\mu_{\mathrm{SS}}$ and $R T_{\mathrm{Base}-\mathrm{SR}}<R T_{\mathrm{Base}-\mathrm{SS}}$ (see Table 2 ), such that sequence information was retrieved more quickly when re-

Table 1

Parameters Used to Model Specific Conditions

\begin{tabular}{llll}
\hline & \multicolumn{3}{c}{ Parameter type } \\
\cline { 2 - 4 } Data and condition & $\mu_{\mathrm{S}}$ & $\mu_{\mathrm{P}}$ & $R T_{\text {Base }}$ \\
\hline Time-course data & & & \\
SS/FS & $\mu_{\mathrm{SS}}$ & $\mu_{\mathrm{P}}$ & $R T_{\text {Base-SS }}$ \\
SS/FR & $\mu_{\mathrm{SS}}$ & $\mu_{\mathrm{P}}$ & $R T_{\text {Base-SS }}$ \\
SR/FS & $\mu_{\mathrm{SR}}$ & $\mu_{\mathrm{P}}$ & $R T_{\text {Base-SR }}$ \\
SR/FR & $\mu_{\mathrm{SCR}}$ & $\mu_{\mathrm{P}}$ & $R T_{\text {Base-SR }}$ \\
Position transition data & & & $R T_{\text {Base-SS }}$ \\
SS/PS/FS & $\mu_{\mathrm{SS}}$ & $\mu_{\mathrm{PS}}$ & $R T_{\text {Base-SS }}$ \\
SS/PS/FR & $\mu_{\mathrm{SS}}$ & $\mu_{\mathrm{PS}}$ & $R T_{\text {Base-SS }}$ \\
SS/PR/FS & $\mu_{\mathrm{SS}}$ & $\mu_{\mathrm{PS}}$ & $R T_{\text {Base-SS }}$ \\
SS/PR/FR & $\mu_{\mathrm{SS}}$ & $\mu_{\mathrm{PS}}$ & $R T_{\text {Base-SR }}$ \\
SR/PS/FS & $\mu_{\mathrm{SR}}$ & $\mu_{\mathrm{PS}}$ & $R T_{\text {Base-SR }}$ \\
SR/PS/FR & $\mu_{\mathrm{SCR}}$ & $\mu_{\mathrm{PS}}$ & $R T_{\text {Base-SR }}$ \\
SR/PR/FS & $\mu_{\mathrm{SR}}$ & $\mu_{\mathrm{PR}}$ & $R T_{\text {Base-SR }}$ \\
SR/PR/FR & $\mu_{\mathrm{SCR}}$ & $\mu_{\mathrm{PCR}}$ & \\
\hline
\end{tabular}

Note. $\mu=$ mean retrieval time; $\mathrm{S}=$ sequence; $\mathrm{P}=$ position; $R T_{\mathrm{Base}}=$ residual processing time; $\mathrm{SS}=$ sequence switch; $\mathrm{SR}=$ sequence repetition; FS = form switch; FR = form repetition; $\mathrm{PS}=$ position switch; $\mathrm{PR}=$ position repetition; $\mathrm{SCR}=$ sequence-cue repetition; $\mathrm{PCR}=$ position-cue repetition. 
Table 2

Best-Fitting Parameter Values From the Model Fit to the Time Course Data

\begin{tabular}{lr}
\hline Parameter & Value (ms) \\
\hline$\mu_{\mathrm{SCR}}$ & 94 \\
$\mu_{\mathrm{SR}}$ & 206 \\
$\mu_{\mathrm{SS}}$ & 302 \\
$\mu_{\mathrm{P}}$ & 806 \\
$R T_{\text {Base-SR }}$ & 885 \\
$R T_{\text {Base-SS }}$ & 1,098 \\
\hline
\end{tabular}

Note. $\mu=$ mean retrieval time; $R T_{\text {Base }}=$ residual processing time; $\mathrm{SCR}=$ sequence-cue repetition; $\mathrm{SR}=$ sequence repetition; $\mathrm{SS}=$ sequence switch; $\mathrm{P}=$ position.

peating a sequence. These benefits decreased with CTI because sequence retrieval can occur during the CTI in Equations 1 and 2, allowing the benefits to be partially absorbed prior to target onset. A nested model in which $\mu_{\mathrm{SR}}$ was constrained to equal $\mu_{\mathrm{SS}}$ and $R T_{\text {Base-SR }}$ was constrained to equal $R T_{\text {Base-SS }}$ fit the data significantly worse than did the full model, $F(2,34)=193.2, p<.001$, with RMSD $=121 \mathrm{~ms}$ and $r=.926$. The model produced a form-repetition benefit only for sequence repetitions (i.e., a cuerepetition benefit) because $\mu_{\mathrm{SCR}}<\mu_{\mathrm{SR}}$, such that the sequence part of the cue was encoded more quickly when its form repeated than when it switched. A nested model in which $\mu_{\mathrm{SCR}}$ was constrained to equal $\mu_{\mathrm{SR}}$ fit the data significantly worse than the full model, $F(1,34)=15.8, p<.001$, with $\mathrm{RMSD}=42 \mathrm{~ms}$ and $r=$ .991. In summary, the assumptions and mechanisms of our model were sufficient to capture several effects from the time course analysis. Although we have reported a fit to group data, we demonstrate in Appendix $\mathrm{C}$ that the model also yields adequate fits to individual subject data, which agree with the fits to the group data.

We next report a fit of the model to the position transition data in Figure 4. Recall that in addition to five parameters used in the previous fit ( $\mu_{\mathrm{SCR}}, \mu_{\mathrm{SR}}, \mu_{\mathrm{SS}}, R T_{\mathrm{Base}-\mathrm{SR}}$, and $\left.R T_{\mathrm{Base}-\mathrm{SS}}\right)$, there were three new parameters associated with different position transitions $\left(\mu_{\mathrm{PCR}}, \mu_{\mathrm{PR}}\right.$, and $\left.\mu_{\mathrm{PS}}\right)$. To impose some constraint on the model fit, we fixed the values of the five old parameters to equal their best-fitting values from the previous fit (see Table 2). Only the three new parameters were free to vary in the model fit to both the sequence and sequence-position cuing data simultaneously (a total of 16 data points). Given that the data in Figure 4 are collapsed across CTI, we used a constant CTI of $720 \mathrm{~ms}$ (the mean of our 5 CTIs) in all equations. All other aspects of the model-fitting procedure remained unchanged. The specific parameters used to model each combination of sequence transition, position transition, and form transition are provided in Table 1.

The model had a good fit to the data, with RMSD $=38 \mathrm{~ms}$ and $r=.993$. The model predictions are plotted as points in Figure 4. The best-fitting values of the new parameters were: $\mu_{\mathrm{PCR}}=605$ $\mathrm{ms}, \mu_{\mathrm{PR}}=716 \mathrm{~ms}$, and $\mu_{\mathrm{PS}}=887 \mathrm{~ms}$ (note that the model predictions in Figure 4 can be generated by plugging these values and the relevant values from Table 2 into Equations 1 and 2 as per the specifications in Table 1, using a CTI of $720 \mathrm{~ms}$ ). The mean $\mu_{P}$ value of $830 \mathrm{~ms}$ from this fit (weighted by transition frequency) is comparable to the best-fitting $\mu_{\mathrm{P}}$ value of $806 \mathrm{~ms}$ from the earlier fit. Most of the model predictions fell within the $95 \%$ confidence intervals plotted around the bars in Figure 4. The only exceptions were position switches for sequence repetitions in the sequenceposition cuing condition, for which the model predictions underestimated the observed data. However, the model captured the overall trends in the data. The model produced position-repetition benefits because $\mu_{\mathrm{PR}}<\mu_{\mathrm{PS}}$, but these were restricted to sequence repetitions because of the structural constraint and tokenspecificity assumptions. A nested model in which $\mu_{P R}$ was constrained to equal $\mu_{\mathrm{PS}}$ fit the data significantly worse than did the full model, $F(1,13)=63.3, p<.001$, with RMSD $=144 \mathrm{~ms}$ and $r=.956$. Position-repetition benefits were amplified by form repetition (more specifically, cue repetition) because $\mu_{\mathrm{PCR}}<\mu_{\mathrm{PR}}$. A nested model in which $\mu_{\mathrm{PCR}}$ was constrained to equal $\mu_{\mathrm{PR}}$ fit the data significantly worse than did the full model, $F(1,13)=8.4$, $p<.05$, with RMSD $=45 \mathrm{~ms}$ and $r=.988$. It is important to note that removing the structural constraint assumption by allowing the same differences in $\mu_{\mathrm{P}}$ for sequence switches that we allowed for sequence repetitions (see the last four rows in the $\mu_{P}$ column of Table 1) also leads to a worse fit, with $\mathrm{RMSD}=70 \mathrm{~ms}$ and $r=$ .962. In summary, the assumptions and mechanisms of our model were sufficient to capture the position transition effects in the data. For reasons discussed in Appendix $\mathrm{C}$, we did not fit the model to individual subject data here.

\section{Summary}

The model was able to capture the major effects in the data (see Figures 3 and 4), reaffirming our conclusion that performance in the sequence-position cuing procedure can be accomplished by retrieving information from a hierarchical representation in memory. Although the primary virtue of the model is its ability to explain a complex pattern of data with only a few assumptions and relatively simple equations, a secondary virtue is the fact that the model provides an interpretable, process-based description of the data with fewer parameters than does a more abstract model such as ANOVA. For example, our model accounted for the data in Figure 3 with only six free parameters, whereas an ANOVA-based account requires 24 parameters (viz. the sum of the numerator degrees of freedom for the nine significant or marginally significant effects depicted in Figure 3), demonstrating that there can be advantages to developing alternatives to linear models (e.g., see Loftus, Oberg, \& Dillon, 2004). More importantly, our model provides a concrete demonstration of how non-isomorphic behavior in the context of the sequence-position cuing procedure can be understood in terms of retrieval from a hierarchically structured representation.

\section{General Discussion}

We found that performance in a novel sequence-position cuing procedure was most consistent with retrieval of information from a hierarchical representation, even though the structure of behavior was not isomorphic to that of the representation. Empirical support for a hierarchical representation included large sequence-repetition benefits, position-repetition benefits only for sequence repetitions, and a lack of robust task-repetition benefits. Theoretical support came from modeling retrieval from a hierarchical representation, whereby we demonstrated that a small set of assumptions and 
equations was sufficient to capture our major empirical effects. Collectively, the methodological, empirical, and theoretical aspects of our work provide deeper insight into the representation of and access to information in memory that comprises a plan for guiding behavior.

\section{Beyond Arbitrary Task Sequences}

As mentioned in the introduction section, we focused on arbitrary task sequences representing non-cumulative plans because they have proved to be informative in recent work (Logan, 2004, 2007; Schneider \& Logan, 2006) and they allowed us to study non-isomorphic behavior. However, it is important to consider how our work could be extended beyond arbitrary task sequences to routine task sequences representing cumulative plans (e.g., plans associated with overarching goals).

The distinction between arbitrary and routine task sequences is not dichotomous; instead, the task sequences exist along a continuum. At one end, there are arbitrary task sequences, which involve relatively independent tasks that are not associated with a predetermined ordering. At the other end, there are routine task sequences, which involve interrelated tasks that are typically associated with only a small subset of all possible orderings. We argue that the transition from one end of the continuum to the otherfrom arbitrary to routine task sequences-occurs as a consequence of learning and practice.

In our experiment, subjects learned that only two orderings of the origin and size tasks were relevant and that those specific task sequences would remain fixed for the duration of the experiment. These constraints allowed subjects to form representations of the task sequences that included ordinal information, such as the position labels in Figure 1. The fidelity of these representations in memory was strengthened by practicing the sequences during the training phase of the experiment, thereby enabling subsequent access to individual items by means of sequence-position cues. Although our task sequences were learned explicitly, we note that ordinal relations among tasks can also be learned implicitly (Gotler, Meiran, \& Tzelgov, 2003; Heuer, Schmidtke, \& Kleinsorge, 2001; Koch, 2001). However, our model does not capture the learning component associated with task sequences because the starting point for the model is the representation that is the end product of learning (e.g., Figure 1A). An important avenue for future research will be to explore how representations of task sequences are constructed during learning (e.g., see Dixon, 1982, 1987, for studies concerning how hierarchical plans are constructed from written directions).

Task sequences become cumulative plans with overarching goals when their constituent tasks become associated with one another beyond an ordinal level-when the tasks become subtasks of a larger task. Typically, these task hierarchies form at a motor level (i.e., as motor programs; see Keele, Cohen, \& Ivry, 1990; Rosenbaum, 1987). For example, when learning to make a cup of coffee, component actions associated with tasks such as adding sugar may become integrated into a motor program. However, task hierarchies may also exist at a semantic level, such that one subtask (adding sugar) becomes associated with another subtask (adding cream) in relation to a higher level task or goal (making the coffee taste better). Applying our model to situations involving task hierarchies may not require changes to the model itself but rather to the representation from which the model retrieves information. A task hierarchy could be represented by adding another level to an existing hierarchical representation (e.g., Figure 1A), with subtask units nested under higher level task units. Modeling task performance would involve accessing the subtask units in a specific order, drawing upon the structural-constraint assumption and partially relaxing the cue-dependency assumption (by allowing subtask units to be accessed without explicit cues; instead, a cue for a higher level task unit would be adequate).

The preceding discussion raises an important difference between our model and those of Botvinick and Plaut (2004) and Cooper and Shallice (2000). In our model, the representation of information concerning a task sequence is separate from the processes that retrieve that information. This can be seen by comparing the hierarchical structure of the representation (Figure 1A) with the non-hierarchical structure of the processes acting on that representation (Equations 1 and 2). In other models, representation and process are intertwined, either in a distributed manner (Botvinick \& Plaut, 2004) or a hierarchical manner (Cooper \& Shallice, 2000). One implication of intertwining representation and process, at least in the modeling of Cooper and Shallice, is that the structure of behavior mirrors the structure of the underlying representation. Whether such isomorphism is necessary is a matter of debate (Botvinick \& Plaut, 2006; Cooper \& Shallice, 2006a, 2006b).

\section{The Value of Modeling}

Breaking the isomorphism between behavior and representation at a methodological level is one thing; doing it at a theoretical level is another. The present study, along with the contrast between the work of Botvinick and Plaut (2004) and Cooper and Shallice (2000), shows how this can be done through modeling. We were able to illustrate in a concrete manner how a hierarchically structured representation could yield non-isomorphic behavior by modeling retrieval from that representation.

The value of modeling is manifest in the present study in several other ways. First, by developing a mathematical model, we were able to (and had to) be explicit about the assumptions underlying our account of the empirical data. Second, by implementing the model, we could verify whether our predictions based on verbal arguments actually followed from those assumptions. Third, we could fit the model to data to see whether those predictions were correct. Fourth, we were able to evaluate the sufficiency and necessity of the model's assumptions and mechanisms by means of nested model fits. By modeling our data, we were able to paint a detailed picture of how information can be retrieved from a hierarchical plan. It is our hope that there will be further attempts to model sequential behavior arising from structured representations, for such efforts are likely to be fruitful in advancing our knowledge of the planning and control of action.

\section{References}

Anderson, J. R., Bothell, D., Lebiere, C., \& Matessa, M. (1998). An integrated theory of list memory. Journal of Memory and Language, 38, 341-380.

Anderson, J. R., \& Matessa, M. (1997). A production system theory of serial memory. Psychological Review, 104, 728-748.

Arrington, C. M., \& Logan, G. D. (2004). Episodic and semantic compo- 
nents of the compound-stimulus strategy in the explicit task-cuing procedure. Memory \& Cognition, 32, 965-978.

Botvinick, M., \& Plaut, D. C. (2004). Doing without schema hierarchies: A recurrent connectionist approach to normal and impaired routine sequential action. Psychological Review, 111, 395-429.

Botvinick, M. M., \& Plaut, D. C. (2006). Such stuff as habits are made on: A reply to Cooper and Shallice (2006). Psychological Review, 113, 917-927.

Collard, R., \& Povel, D.-J. (1982). Theory of serial pattern production: Tree traversals. Psychological Review, 89, 693-707.

Collins, A. M., \& Quillian, M. R. (1969). Retrieval time from semantic memory. Journal of Verbal Learning and Verbal Behavior, 8, $240-$ 247.

Cooper, R., \& Shallice, T. (2000). Contention scheduling and the control of routine activities. Cognitive Neuropsychology, 17, 297-338.

Cooper, R. P., \& Shallice, T. (2006a). Hierarchical schemas and goals in the control of sequential behavior. Psychological Review, 113, 887-916.

Cooper, R. P., \& Shallice, T. (2006b). Structured representations in the control of behavior cannot be so easily dismissed: A reply to Botvinick and Plaut (2006). Psychological Review, 113, 929-931.

De Jong, R. (1995). The role of preparation in overlapping-task performance. Quarterly Journal of Experimental Psychology, 48A, 2-25.

Dixon, P. (1982). Plans and written directions for complex tasks. Journal of Verbal Learning and Verbal Behavior, 21, 70-84

Dixon, P. (1987). The structure of mental plans for following directions. Journal of Experimental Psychology: Learning, Memory, and Cognition, 13, 18-26.

Duncan, J. (1986). Disorganisation of behaviour after frontal lobe damage. Cognitive Neuropsychology, 3, 273-290.

Gillund, G., \& Shiffrin, R. M. (1984). A retrieval model for both recognition and recall. Psychological Review, 91, 1-67.

Gotler, A., Meiran, N., \& Tzelgov, J. (2003). Nonintentional task set activation: Evidence from implicit task sequence learning. Psychonomic Bulletin \& Review, 10, 890-896.

Greeno, J. G., \& Simon, H. A. (1974). Processes for sequence production. Psychological Review, 81, 187-198.

Heuer, H., Schmidtke, V., \& Kleinsorge, T. (2001). Implicit learning of sequences of tasks. Journal of Experimental Psychology: Learning, Memory, and Cognition, 27, 967-983.

Hintzman, D. L. (1986). "Schema abstraction" in a multiple-trace memory model. Psychological Review, 93, 411-428.

Humphreys, G. W., \& Forde, E. M. E. (1998). Disordered action schema and action disorganisation syndrome. Cognitive Neuropsychology, 15, $771-811$.

Johnson, N. F. (1970). The role of chunking and organization in the process of recall. In G. H. Bower (Ed.), The psychology of learning and motivation (Vol. 4, pp. 171-247). New York: Academic Press.

Keele, S. W., Cohen, A., \& Ivry, R. (1990). Motor programs: Concepts and issues. In M. Jeannerod (Ed.), Attention and performance XIII: Motor representation and control (pp. 77-110). Hillsdale, NJ: Erlbaum.

Koch, I. (2001). Automatic and intentional activation of task sets. Journal of Experimental Psychology: Learning, Memory, and Cognition, 27, $1474-1486$.

Lashley, K. S. (1951). The problem of serial order in behavior. In L. A. Jeffress (Ed.), Cerebral mechanisms in behavior (pp. 112-136). New York: Wiley.

Lien, M.-C., \& Ruthruff, E. (2004). Task switching in a hierarchical task structure: Evidence for the fragility of the task repetition benefit. Journal of Experimental Psychology: Learning, Memory, and Cognition, 30, 697-713

Loftus, G. R., Oberg, M. A., \& Dillon, A. M. (2004). Linear theory, dimensional theory, and the face-inversion effect. Psychological Review, $111,835-863$.

Logan, G. D. (2004). Working memory, task switching, and executive control in the task span procedure. Journal of Experimental Psychology: General, 133, 218-236.

Logan, G. D. (2007). What it costs to implement a plan: Plan-level and task-level contributions to switch costs. Memory \& Cognition, 35, 591602

Logan, G. D., \& Bundesen, C. (2003). Clever homunculus: Is there an endogenous act of control in the explicit task-cuing procedure? Journal of Experimental Psychology: Human Perception and Performance, 29, $575-599$.

Logan, G. D., \& Bundesen, C. (2004). Very clever homunculus: Compound stimulus strategies for the explicit task-cuing procedure. Psychonomic Bulletin \& Review, 11, 832-840.

Logan, G. D., \& Schneider, D. W. (2006). Priming or executive control? Associative priming of cue encoding increases "switch costs" in the explicit task-cuing procedure. Memory \& Cognition, 34, 1250-1259.

Logan, G. D., Schneider, D. W., \& Bundesen, C. (2007). Still clever after all these years: Searching for the homunculus in explicitly cued task switching. Journal of Experimental Psychology: Human Perception and Performance, 33, 978-994.

Luria, R., \& Meiran, N. (2003). Online order control in the psychological refractory period paradigm. Journal of Experimental Psychology: Human Perception and Performance, 29, 556-574.

Mayr, U., \& Kliegl, R. (2003). Differential effects of cue changes and task changes on task-set selection costs. Journal of Experimental Psychology: Learning, Memory, and Cognition, 29, 362-372.

McClelland, J. L. (1979). On the time relations of mental processes: An examination of systems of processes in cascade. Psychological Review, 86, 287-330.

Miller, G. A., Galanter, E., \& Pribram, K. H. (1960). Plans and the structure of behavior. New York: Holt, Rinehart, \& Winston.

Monsell, S. (2003). Task switching. Trends in Cognitive Sciences, 7 , $134-140$.

Morris, R., \& Ward, G. (Eds.). (2005). The cognitive psychology of planning. Hove, United Kingdom: Psychology Press.

Povel, D.-J., \& Collard, R. (1982). Structural factors in patterned finger tapping. Acta Psychologica, 52, 107-123.

Ratcliff, R. (1978). A theory of memory retrieval. Psychological Review, 85, 59-108.

Ratcliff, R., \& McKoon, G. (1988). A retrieval theory of priming in memory. Psychological Review, 95, 385-408.

Rosenbaum, D. A. (1987). Hierarchical organization of motor programs. In S. Wise (Ed.), Neural and behavioral approaches to higher brain functions (pp. 45-66). New York: Wiley.

Rosenbaum, D. A., Kenny, S. B., \& Derr, M. A. (1983). Hierarchical control of rapid movement sequences. Journal of Experimental Psychology: Human Perception and Performance, 9, 86-102.

Schank, R. C., \& Abelson, R. P. (1977). Scripts, plans, goals, and understanding. Hillsdale, NJ: Erlbaum.

Schneider, D. W., \& Logan, G. D. (2005). Modeling task switching without switching tasks: A short-term priming account of explicitly cued performance. Journal of Experimental Psychology: General, 134, 343-367.

Schneider, D. W., \& Logan, G. D. (2006). Hierarchical control of cognitive processes: Switching tasks in sequences. Journal of Experimental Psychology: General, 135, 623-640.

Schneider, D. W., \& Logan, G. D. (2007). Task switching versus cue switching: Using transition cuing to disentangle sequential effects in task-switching performance. Journal of Experimental Psychology: Learning, Memory, and Cognition, 33, 370-378.

Tulving, E., \& Thomson, D. M. (1973). Encoding specificity and retrieval processes in episodic memory. Psychological Review, 80, 352-373. 


\section{Appendix A}

Mean Response Time (RT, in Milliseconds) and Error Rate (ER, as Percentage of Incorrect Responses) in Each Cuing Condition as a Function of Sequence Transition, Task Transition,

Form Transition, and Cue-Target Interval (in Milliseconds)

\begin{tabular}{|c|c|c|c|c|c|c|c|c|}
\hline \multirow{2}{*}{$\begin{array}{l}\text { Sequence } \\
\text { transition }\end{array}$} & \multirow{2}{*}{$\begin{array}{c}\text { Task } \\
\text { transition }\end{array}$} & \multirow{2}{*}{$\begin{array}{c}\text { Form } \\
\text { transition }\end{array}$} & \multirow[b]{2}{*}{ Measure } & \multicolumn{5}{|c|}{ Cue-target interval } \\
\hline & & & & $0 \mathrm{~ms}$ & $300 \mathrm{~ms}$ & $700 \mathrm{~ms}$ & $1,100 \mathrm{~ms}$ & $1,500 \mathrm{~ms}$ \\
\hline \multicolumn{9}{|c|}{ Sequence cuing condition } \\
\hline \multirow[t]{2}{*}{ SS } & TS & FS & RT & 2,170 & 2,082 & 1,991 & 1,922 & 1,875 \\
\hline & & & ER & 2.2 & 2.8 & 3.5 & 3.1 & 2.7 \\
\hline \multirow[t]{2}{*}{ SS } & TS & FR & RT & 2,104 & 2,049 & 1,882 & 1,930 & 1,938 \\
\hline & & & ER & 2.4 & 3.8 & 4.3 & 3.3 & 3.6 \\
\hline \multirow[t]{2}{*}{ SS } & TR & FS & RT & 2,213 & 2,011 & 1,982 & 1,906 & 1,817 \\
\hline & & & ER & 2.5 & 2.1 & 2.5 & 1.2 & 2.6 \\
\hline \multirow[t]{2}{*}{ SS } & TR & FR & RT & 2,206 & 2,117 & 1,999 & 1,991 & 1,870 \\
\hline & & & ER & 3.3 & 2.1 & 2.7 & 1.3 & 3.6 \\
\hline \multirow[t]{2}{*}{ SR } & TS & FS & RT & 1,951 & 1,829 & 1,812 & 1,795 & 1,737 \\
\hline & & & ER & 4.1 & 4.2 & 5.3 & 5.0 & 3.1 \\
\hline \multirow{2}{*}{ SR } & TS & FR & RT & 1,872 & 1,828 & 1,788 & 1,717 & 1,662 \\
\hline & & & ER & 3.7 & 4.8 & 6.2 & 3.5 & 4.3 \\
\hline \multirow[t]{2}{*}{ SR } & $\mathrm{TR}$ & FS & RT & 1,800 & 1,556 & 1,617 & 1,717 & 1,643 \\
\hline & & & ER & 3.0 & 2.6 & 1.3 & 2.2 & 2.6 \\
\hline \multirow[t]{2}{*}{ SR } & TR & FR & $\mathrm{RT}$ & 1,642 & 1,592 & 1,588 & 1,539 & 1,619 \\
\hline & & & ER & 1.5 & 2.4 & 3.4 & 3.8 & 2.8 \\
\hline \multicolumn{9}{|c|}{ Sequence-position cuing condition } \\
\hline \multirow[t]{2}{*}{ SS } & TS & FS & RT & 2,159 & 1,921 & 1,590 & 1,425 & 1,244 \\
\hline & & & ER & 4.2 & 2.9 & 4.5 & 0.7 & 3.3 \\
\hline \multirow[t]{2}{*}{ SS } & TS & FR & RT & 2,251 & 1,958 & 1,588 & 1,390 & 1,274 \\
\hline & & & ER & 3.2 & 2.4 & 2.9 & 1.9 & 3.1 \\
\hline \multirow[t]{2}{*}{ SS } & TR & FS & RT & 2,232 & 1,934 & 1,701 & 1,400 & 1,318 \\
\hline & & & ER & 3.1 & 2.6 & 2.2 & 2.2 & 2.4 \\
\hline \multirow{2}{*}{ SS } & TR & FR & RT & 2,170 & 1,927 & 1,604 & 1,329 & 1,292 \\
\hline & & & ER & 3.3 & 3.2 & 2.4 & 2.1 & 2.4 \\
\hline \multirow[t]{2}{*}{ SR } & TS & FS & RT & 2,097 & 1,681 & 1,435 & 1,226 & 1,157 \\
\hline & & & ER & 2.4 & 3.5 & 2.7 & 2.4 & 2.8 \\
\hline \multirow[t]{2}{*}{ SR } & TS & FR & RT & 1,978 & 1,647 & 1,318 & 1,189 & 1,066 \\
\hline & & & ER & 3.7 & 3.0 & 1.9 & 3.7 & 3.0 \\
\hline \multirow[t]{2}{*}{ SR } & TR & FS & RT & 1,826 & 1,483 & 1,197 & 1,135 & 1,049 \\
\hline & & & ER & 2.1 & 3.4 & 2.3 & 1.6 & 0.9 \\
\hline \multirow[t]{2}{*}{ SR } & TR & FR & RT & 1,651 & 1,360 & 1,210 & 1,077 & 994 \\
\hline & & & ER & 2.6 & 2.5 & 2.0 & 3.4 & 1.9 \\
\hline
\end{tabular}

Note. $\mathrm{SS}=$ sequence switch; $\mathrm{SR}=$ sequence repetition; $\mathrm{TS}=$ task switch; $\mathrm{TR}=$ task repetition; $\mathrm{FS}=$ form switch; $\mathrm{FR}=$ form repetition. 


\section{Appendix B}

Summary for the Analyses of Variance Conducted on Mean Response Time and Error Rate as a Function of Cuing Condition, Sequence Transition, Task Transition, Form Transition, and CueTarget Interval (CTI)

\begin{tabular}{|c|c|c|c|c|c|c|c|}
\hline \multirow[b]{2}{*}{ Effect } & \multirow[b]{2}{*}{$d f$} & \multicolumn{3}{|c|}{ Response time } & \multicolumn{3}{|c|}{ Error rate } \\
\hline & & $F$ & MSE & $\eta_{P}^{2}$ & $F$ & $M S E$ & $\eta_{P}^{2}$ \\
\hline Cuing condition (C) & 1,62 & $18.8^{* *}$ & $3,527,161$ & .23 & 0.8 & 165 & .01 \\
\hline Sequence transition $(\mathrm{S})$ & 1,62 & $287.3^{* *}$ & 190,088 & .82 & 1.9 & 23 & .03 \\
\hline Task transition $(\mathrm{T})$ & 1,62 & $39.3^{* *}$ & 105,186 & .39 & $21.9^{* * *}$ & 23 & .26 \\
\hline Form transition $(\mathrm{F})$ & 1,62 & $12.7^{* * *}$ & 64,552 & .17 & 2.5 & 17 & .04 \\
\hline Cue-target interval (I) & 4,248 & $247.5^{* * *}$ & 100,545 & .80 & 1.1 & 21 & .02 \\
\hline $\mathrm{C} \times \mathrm{S}$ & 1,62 & 0.1 & 190,088 & .00 & $5.4^{*}$ & 23 & .08 \\
\hline $\mathrm{C} \times \mathrm{T}$ & 1,62 & 0.1 & 105,186 & .00 & $4.8^{*}$ & 23 & .07 \\
\hline $\mathrm{C} \times \mathrm{F}$ & 1,62 & 1.2 & 64,552 & .02 & 0.9 & 17 & .01 \\
\hline $\mathrm{C} \times \mathrm{I}$ & 4,248 & $94.7^{* *}$ & 100,545 & .60 & 1.5 & 21 & .02 \\
\hline $\mathrm{S} \times \mathrm{T}$ & 1,62 & $64.3^{* *}$ & 88,543 & .51 & $3.2^{\dagger}$ & 23 & .05 \\
\hline $\mathrm{S} \times \mathrm{F}$ & 1,62 & $15.4^{* *}$ & 49,437 & .20 & 0.2 & 16 & .00 \\
\hline $\mathrm{S} \times \mathrm{I}$ & 4,248 & $11.1^{* *}$ & 53,547 & .15 & $2.5^{*}$ & 20 & .04 \\
\hline $\mathrm{T} \times \mathrm{F}$ & 1,62 & 0.1 & 35,312 & .00 & 0.3 & 22 & .00 \\
\hline $\mathrm{T} \times \mathrm{I}$ & 4,248 & $2.6^{*}$ & 58,464 & .04 & 1.0 & 19 & .02 \\
\hline $\mathrm{F} \times \mathrm{I}$ & 4,248 & $2.0^{\dagger}$ & 48,000 & .03 & 0.5 & 21 & .01 \\
\hline $\mathrm{C} \times \mathrm{S} \times \mathrm{T}$ & 1,62 & 0.0 & 88,543 & .00 & 1.1 & 23 & .02 \\
\hline $\mathrm{C} \times \mathrm{S} \times \mathrm{F}$ & 1,62 & 0.0 & 49,437 & .00 & 1.1 & 16 & .02 \\
\hline $\mathrm{C} \times \mathrm{S} \times \mathrm{I}$ & 4,248 & 1.1 & 53,547 & .02 & 0.4 & 20 & .01 \\
\hline $\mathrm{C} \times \mathrm{T} \times \mathrm{F}$ & 1,62 & $5.3^{*}$ & 35,312 & .08 & 0.1 & 22 & .00 \\
\hline $\mathrm{C} \times \mathrm{T} \times \mathrm{I}$ & 4,248 & 1.5 & 58,464 & .02 & $2.0^{\dagger}$ & 19 & .03 \\
\hline $\mathrm{C} \times \mathrm{F} \times \mathrm{I}$ & 4,248 & 0.5 & 48,000 & .01 & 1.5 & 21 & .02 \\
\hline $\mathrm{S} \times \mathrm{T} \times \mathrm{F}$ & 1,62 & 0.1 & 51,192 & .00 & 0.0 & 25 & .00 \\
\hline $\mathrm{S} \times \mathrm{T} \times \mathrm{I}$ & 4,248 & $4.7^{*}$ & 60,091 & .07 & 0.1 & 22 & .00 \\
\hline $\mathrm{S} \times \mathrm{F} \times \mathrm{I}$ & 4,248 & 1.7 & 44,046 & .03 & 0.4 & 20 & .01 \\
\hline $\mathrm{T} \times \mathrm{F} \times \mathrm{I}$ & 4,248 & 0.9 & 48,830 & .01 & 0.3 & 20 & .01 \\
\hline $\mathrm{C} \times \mathrm{S} \times \mathrm{T} \times \mathrm{F}$ & 1,62 & $5.9^{*}$ & 51,192 & .09 & 0.3 & 25 & .01 \\
\hline $\mathrm{C} \times \mathrm{S} \times \mathrm{T} \times \mathrm{I}$ & 4,248 & 0.9 & 60,091 & .01 & 1.5 & 22 & .02 \\
\hline $\mathrm{C} \times \mathrm{S} \times \mathrm{F} \times \mathrm{I}$ & 4,248 & $2.1^{\dagger}$ & 44,046 & .03 & 0.8 & 20 & .01 \\
\hline $\mathrm{C} \times \mathrm{T} \times \mathrm{F} \times \mathrm{I}$ & 4,248 & 0.5 & 48,830 & .01 & 0.6 & 20 & .01 \\
\hline $\mathrm{S} \times \mathrm{T} \times \mathrm{F} \times \mathrm{I}$ & 4,248 & 0.9 & 44,356 & .01 & 1.1 & 19 & .02 \\
\hline $\mathrm{C} \times \mathrm{S} \times \mathrm{T} \times \mathrm{F} \times \mathrm{I}$ & 4,248 & 0.8 & 44,356 & .01 & 0.3 & 19 & .01 \\
\hline
\end{tabular}

${ }^{\dagger} p<.10 .{ }^{*} p<.05 .{ }^{* *} p<.001$.

\section{Appendix C}

Results of Additional Model Fits to Group Data and Individual Subject Data for the Time Course Results in Figure 3

In this appendix, we present the results of additional model fits to group data and to individual subject data for the time course results presented in Figure 3. To facilitate comparison of the parameter values across group and individual fits, we performed separate fits to the group data for each cuing condition, such that the parameter values used to fit the sequence cuing condition were not constrained to equal those used to fit the sequence-position cuing condition, unlike in the simultaneous fit to both cuing conditions reported in the main text. Consequently, each group fit yields parameter values that are based on data from a single cuing condition, as is the case with the individual fits (by necessity, because cuing condition is a between-subjects variable).

We fit the sequence-position cuing condition using Equation 2, and we fit the sequence cuing condition using a reduced form of
Equation 1. We could not apply Equation 1 directly to the sequence cuing condition because when the parameter values are not constrained to match those used in Equation 2, $R T_{\text {Base }}$ and $\mu_{\mathrm{P}}$ are no longer separately identifiable because they are additive terms in Equation 1. Consequently, one cannot obtain unique estimates for each parameter if Equation 1 is applied in isolation to the sequence cuing data. For this reason, we allowed $\mu_{\mathrm{P}}$ to be subsumed in $R T_{\text {Base }}$, resulting in a reduced form of Equation 1 with one less parameter:

$$
R T=R T_{\text {Base }}+\mu_{\mathrm{S}} \cdot \exp \left(-\mathrm{CTI} / \mu_{\mathrm{S}}\right) .
$$

All other aspects of the model fitting were the same as before. There were five free parameters $\left(\mu_{\mathrm{SCR}}, \mu_{\mathrm{SR}}, \mu_{\mathrm{SS}}, R T_{\mathrm{Base}-\mathrm{SR}}\right.$, and $R T_{\text {Base-ss }}$ ) for fitting the 20 data points in the sequence cuing condition and six free parameters (same as above, plus $\mu_{\mathrm{P}}$ ) for 
fitting the 20 data points in the sequence-position cuing condition. As a constraint on the fits, we forced $\mu_{\mathrm{SCR}} \leq \mu_{\mathrm{SR}} \leq \mu_{\mathrm{SS}}$ and $R T_{\text {Base-Ss }} \leq R T_{\text {Base-SR }}$, which is the pattern observed in the earlier fits.

The results of the group and individual fits were generally in good agreement. For the sequence cuing condition, the group fit yielded RMSD $=38 \mathrm{~ms}$ and $r=.975$, and the individual fits yielded mean values of RMSD $=139 \mathrm{~ms}$ and $r=.761$. The higher RMSD value and lower $r$ value for the individual fits is to be expected because each individual data pattern is much noisier than the group data pattern; the former is based on about 1/32 (i.e., $1 / N$ ) the number of observations per cell in the design. The best-fitting parameter values for each fit are provided in Table $\mathrm{C} 1$. The group parameter values all fell within the $95 \%$ confidence intervals around the mean individual parameter values, suggesting no substantial discrepancies between the group and individual fits. Moreover, the predicted RTs from the group fit were similar to the averages of the predicted RTs from the individual fits (RMSD = $18 \mathrm{~ms}$ and $r=.995$ ).

For the sequence-position cuing condition, the group fit yielded RMSD $=23 \mathrm{~ms}$ and $r=.998$, and the individual fits yielded mean values of RMSD $=132 \mathrm{~ms}$ and $r=.931$. The higher mean $r$ value for individual fits to this condition compared with the sequence cuing condition (.931 vs. .761) is likely due to differences in the steepness of the time-course functions. High within-subject variability can lead to non-monotonic time-course functions, especially if the functions are shallow, as in the sequence cuing condition (see Figure 3). Given that the model predicts monotonic time-course functions, any deviations from monotonicity in the data will reduce the correlation between model and data, as we observed in the sequence cuing condition. The steeper time-course functions in the sequence-position cuing condition are less affected by noise (i.e., deviations from monotonicity are less likely), resulting in a higher correlation. The best fitting parameter values for each fit are provided in Table $\mathrm{C} 1$. With the exception of $R T_{\text {Base-SR, }}$

Table C1

Best Fitting Parameter Values (in Milliseconds) From the Group and Individual Model Fits to the Time-Course Data

\begin{tabular}{lccccc}
\hline & \multicolumn{2}{c}{ Sequence cuing } & & \multicolumn{2}{c}{$\begin{array}{c}\text { Sequence-position } \\
\text { cuing }\end{array}$} \\
\cline { 2 - 3 } \cline { 5 - 6 } Parameter & Group & Individual & & Group & Individual \\
\cline { 2 - 3 } & 73 & $135 \pm 72$ & & 352 & $364 \pm 145$ \\
$\mu_{\mathrm{SCR}}$ & 179 & $212 \pm 85$ & & 459 & $472 \pm 148$ \\
$\mu_{\mathrm{SR}}$ & 297 & $351 \pm 117$ & & 671 & $657 \pm 134$ \\
$\mu_{\mathrm{SS}}$ & & & & 457 & $566 \pm 124$ \\
$\mu_{\mathrm{P}}$ & 1,686 & $1,646 \pm 96$ & & 992 & $865 \pm 106$ \\
$R T_{\text {Base-SR }}$ & 1,914 & $1,851 \pm 134$ & & 1,085 & $982 \pm 115$ \\
$R T_{\text {Base-SS }}$ & & & & &
\end{tabular}

Note. $\mu=$ mean retrieval time; $R T_{\text {Base }}=$ residual processing time; $\mathrm{SCR}=$ sequence-cue repetition; $\mathrm{SR}=$ sequence repetition; $\mathrm{SS}=$ sequence switch; $\mathrm{P}=$ position. Group parameter values were derived from separate fits to each cuing condition, so they are not identical to the values in Table 2 . Individual parameter values are means of parameter values ( \pm values for the $95 \%$ confidence intervals) from fits to individual participants in each cuing condition. For the sequence cuing condition, $\mu_{\mathrm{P}}$ is subsumed in $R T_{\text {Base }}$ (see text for details). the group parameter values all fell within the $95 \%$ confidence intervals around the mean individual parameter values, suggesting almost no discrepancies between the group and individual fits. Moreover, the predicted RTs from the group fit were very similar to the averages of the predicted RTs from the individual fits $(\mathrm{RMSD}=9 \mathrm{~ms}$ and $r=.9998)$.

The agreement between the group and individual fits indicates that the patterns of data and corresponding model fits presented in Figure 3 do not reflect averaging artifacts. Thus, the high quality of the earlier fits derives from fitting data that were generally consistent across subjects, but for which within-subject variability or noise (which is presumably idiosyncratic and uncorrelated across subjects) was averaged out.

For a set of interrelated reasons, we did not perform additional model fits to group data and to individual subject data for the position transition results in Figure 4. In our earlier fits to that data, the parameters associated with sequence transitions $\left(\mu_{\mathrm{SCR}}, \mu_{\mathrm{SR}}\right.$, $\mu_{\mathrm{SS}}, R T_{\mathrm{Base}-\mathrm{SR}}$, and $\left.R T_{\mathrm{Base}-\mathrm{SS}}\right)$ were set to the best-fitting values from the fit to the time course data in Figure 3, so only parameters associated with position transitions $\left(\mu_{\mathrm{PCR}}, \mu_{\mathrm{PR}}\right.$, and $\left.\mu_{\mathrm{PS}}\right)$ were free to vary. An issue with using the same approach for individual fits is that any noise-related irregularities (e.g., non-monotonicities) in the time-course functions from the individual fits described above would affect the estimates of the parameters that would be fixed for fitting the position transition data. Thus, a fixed parameter value may be suboptimal for subsequent model fitting at the outset, making it unclear whether a poor fit to the position transition data would reflect a limitation of the model or instead arise from using suboptimal values for the fixed parameters. This is not a serious issue with our earlier fits because within-subject variability is averaged out in the group data and thus the estimated parameter values from one fit may be close to optimal for use in another fit

One way to circumvent this issue is to allow all eight parameters to be free to vary when fitting the position transition data. However, this approach is not feasible in the present context because then the model becomes underconstrained, such that it can make perfect predictions for all of the sequence-repetition conditions. An alternative approach is to fit only the sequence-switch conditions, for which the model makes the same prediction for all combinations of position transition and form transition. However, the single value that minimizes RMSD is simply the mean of the $N$ data points, so RMSD is directly related to their variance $(V A R)$ :

$$
R M S D=\sqrt{\frac{(N-1) V A R}{N}} .
$$

Increased within-subject variability is guaranteed to increase RMSD, and when the number of data points per subject is small (in the present context, $N=4$ ), a single aberrant observation can impair the fit substantially. Moreover, $r$ cannot be calculated under these circumstances because there is no variance in the model predictions. In consideration of these issues, individual fits to the position transition data were deemed to be unfeasible.

Received February 28, 2007

Revision received June 26, 2007

Accepted July 3, 2007 